\title{
Underwater Low-Frequency Sound Absorbing Metamaterials Based on Wave-Mode Transformation and Local Resonance Mechanism
}

\author{
Han Zhang ${ }^{a, b, c, *}$, Pengxiang $\mathrm{HaO}^{c}, \mathrm{Yang}_{\mathrm{GaO}}{ }^{d}$, Yong $\mathrm{ChenG}^{e}$, \\ Guangzeng Li ${ }^{f}$, Ning HU ${ }^{c}$ And ZhengPan QI ${ }^{c}$ \\ ${ }^{a}$ Key Laboratory of Noise and Vibration, Institute of Acoustics, \\ Chinese Academy of Sciences, 100190, Beijing, China \\ ${ }^{b}$ University of Chinese Academy of Sciences, 100049, Beijing, China \\ ${ }^{c}$ Hebei University of Technology, 300401, Tianjin, China \\ ${ }^{d}$ College of Science, China Agricultural University, 100083, Beijing, China \\ ${ }^{e}$ Key Laboratory of Mechanical Reliability for Heavy Equipments and Large Structures of Hebei \\ Province, Yanshan University, 066004, Qinhuangdao, China \\ ${ }^{f}$ Institute of Mechanics, Chinese Academy of Sciences, 100190, Beijing, China
}

Received: 02.10.2021 \& Accepted: 08.11.2021

Doi: 10.12693/APhysPolA.140.455

*e-mail: zhanghan@mail.ioa.ac.cn

\begin{abstract}
We present a low-frequency sound-absorbing metamaterial for underwater applications and verify that its sound-absorbing coefficient reaches above 0.7 within 45 to $300 \mathrm{~Hz}$ bandwidth and remains more than 0.9 within 110 to $300 \mathrm{~Hz}$ bandwidth by finite element method. The metamaterial unit structure consists of five groups of conical and cylindrical cavities, a rubber matrix, two metal vibrators and a steel backing. We first analyze that the high sound absorption performance of this structure at low-frequency is the result of two mechanisms of local resonance and wave-mode transformation. The finite element method results prove good sound absorption performance of the structure and explain the influence of changing the geometrical parameters of the structure on the sound absorption coefficient. The combined effect of the local resonance, wave-mode transformation and sound wave scattering mechanism made a great contribution to the structure's high sound absorption efficiency. The innovative design propose in this study has potential value in the research and application fields of underwater sound absorption.

topics: sound-absorbing metamaterials, wave-mode transformation, local resonance, low-frequency sound absorption
\end{abstract}

\section{Introduction}

Polymer viscoelastic materials such as butyl rubber [1-3], polyurethane rubber $[4,5]$, interpenetrating polymer network $[6,7]$ etc., have been always the preferred materials for the design of underwater sound-absorbing structures due to their high internal friction and good damping performance [8]. For the ideal sound-absorbing material, on the one hand, its impedance must match the interface impedance of the water medium to minimize the primary reflection wave; on the other hand, it needs to have a high loss of sound waves inside the structure, so that the sound wave energy entering the covering layer can be attenuated as far as possible to achieve high performance sound absorption. However, uniform viscoelastic materials cannot achieve high impedance matching and high acoustic energy loss at the same time [9], and the relaxation absorption is far from meeting the needs of various engineering applications. Obviously, it becomes necessary to reasonably design the acoustic structure inside the sound-absorbing material in order not only to achieve impedance matching with water, but also enhance the attenuation effect of the incident plane waves through mechanisms such as wave-mode transformation and local resonance. Many studies have shown that embedding scatterers (heterogeneous media or obstacles) in the base material is an effective way to realize wave-mode transformation [8]. When the $P$-wave incident in the covering layer contacts the scatterer, a wavemode transformation will occur in the side direction of the scatterer, part of the $P$-wave will be transformed into a shear wave with a higher loss factor, and then absorbed by the viscoelastic material. In recent years, a lot of research has been carried out on this kind of structure, among which the cavity resonant sound absorption structure has attracted special attention because of its excellent sound absorption performance in the low-frequency range $[10,11]$. 
Cavity resonant sound-absorbing material is a kind of underwater acoustic functional material made by introducing spherical and cylindrical cavity structures into the uniform sound-absorbing material [12]. These materials realize the effective absorption of sound waves through the mechanism of local resonance, wave-mode transformation and combining with the intrinsic characteristics of high polymer viscoelastic materials. The application of cavity resonant sound absorbing material can be traced back to the "Aiberich" anechoic tile on the conventional submarine in the 1940s, which installed a layer of synthetic rubber sound absorbing body on the hull of the submarine and absorbed the sound energy by the cavity resonant generated when the acoustic wave incident. Since then, a lot of research have been carried out on the cavity resonance type sound absorption structure and its sound absorption mechanism. In addition, the periodic scatterers structure has been proved to have special acoustic scattering characteristicsband gap characteristics [13, 14]. Tao [15] used the principle of local resonance combined with wavemode transformation to embed periodic scattered particles in the matrix material. Through the heavy particles wrapped in the soft material to realize the wave conversion during the scattering and resonance of the acoustic wave, so as to improve the acoustic absorption ability of the covering layer. In recent years, Sharma et al. [16] has designed a digital model of underwater sound-absorbing covering layer and performed simulation analysis with polydimethylsiloxane (PDMS) as the matrix and embedded cylindrical cavity in the horizontal direction. The sound absorption characteristics of the covering layer under different parameter conditions were explored. In [3], Sharma's team used rubber as the matrix material, embedded the cylindrical cavity and steel column layer in the horizontal direction, and the sound absorption performance was greatly improved in the range of $2-5 \mathrm{kHz}$. Its average sound absorption coefficient reaches 0.6 , but the effect is poor in the range below $2 \mathrm{kHz}$. Zhao et al. [17] proposed the idea of a two-layer "Alberich" structure and used the differential evolution algorithm to optimize the parameters of the two-layer structure. It has good sound absorption performance in the range of more than $2-10 \mathrm{kHz}$, and the average sound absorption coefficient is about 0.8 . In addition, Gao and $\mathrm{Lu}$ [1] proposed a periodic underwater sound absorption structure with rubber as the matrix and metal blocks embedded in the cavity. Through calculation and simulation, it was verified that the sound absorption coefficient of the structure was greater than 0.4 in the range of $240 \mathrm{~Hz}^{-}$ $10 \mathrm{kHz}$. On this basis, Gao's team [18] designed a structure containing two metal disks, achieving a sound absorption coefficient greater than 0.5 in the range of 50-300 Hz. Although these novel design methods and corresponding experiments provide a wealth of ideas for underwater sound-absorbing coatings, their sound absorption frequency range is still dominated by medium and high frequencies, so low-frequency sound absorption performance is poor and unstable. As the requirements of lowfrequency sound absorption conditions are not met, it is difficult to promote and apply underwater sound-absorbing coatings in actual work.

The sound absorption process of the underwater sound absorption structure can be regarded as the process in which the sound waves in water pass through the surface of the water/sound absorption material, enter inside and are absorbed by the material. Therefore, this paper presents a kind of underwater sound-absorbing metamaterial based on wave-mode transformation and local resonance principle, which can minimize the primary reflection wave and improve the absorption performance of low-frequency sound waves. The metamaterial is a polymer viscoelastic material, in which a conical and cylindrical cavity and two metal vibrators are embedded. The sound absorption performance of the metamaterial is verified by finite element method. Furthermore, the influence of the parameters on the performance of sound absorption is discussed. The conclusions will provide a powerful theoretical and design basis for research of underwater low-frequency sound absorption project.

\section{Description of sound absorption mechanism}

Since Zwikker and Kosten introduced an analysis of the sound absorption mechanism of underwater sound-absorbing coatings in "Sound absorbing materials" in 1949 [19], the sound absorption mechanism of sound-absorbing materials has always attracted a lot of attention, including cavity resonance absorption. The sound absorption mechanism of acoustic structures is currently a hot research topic [20, 21]. Regarding the metamaterial structure in this paper, it is formed by adding a multilayer cone, a cylindrical cavity, and two metal blocks as vibrators to the uniform viscoelastic rubber, as shown in Fig. 1. The focus of the research is mainly on sound wave scattering, local resonance absorption and wave mode conversion [22].

First of all, the radius of the gradient of the upper conical cavity shows that the equivalent impedance is gradually changing, as shown in Fig. 1a and b. This is also the application of impedance gradient structure in underwater sound absorption. When plane waves are incident perpendicularly to the metamaterial from water, air in the cavity as a fluid medium only allows the $P$-wave to propagate. In addition, the reflection of sound waves on the interface of the impedance gradient structure is only related to the incident angle of the sound wave and the Poisson ratio of the rubber matrix, irrespective of frequency [1]. Therefore, the inclined interface causes the elastic wave in the rubber matrix to change during the propagation process. By changing the 

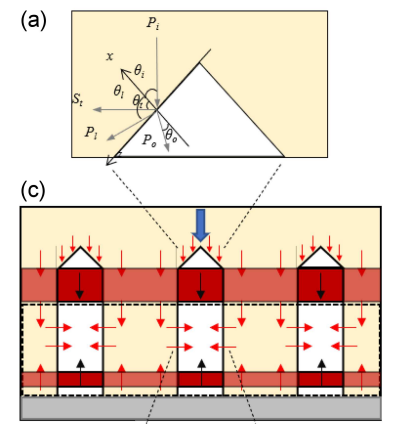

(e)

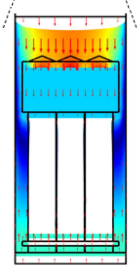

(b)

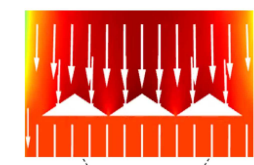

(d)

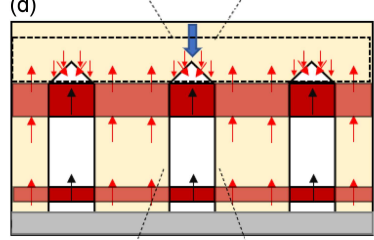

(f)

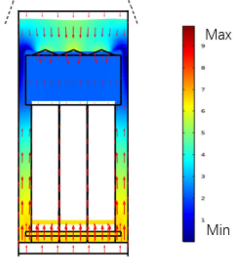

Fig. 1. Sound absorption mechanism diagram.

propagation direction, the reflection of sound waves at the interface between water and material is reduced, and continuous reflection and scattering occur, which improves the sound absorption effect in low-frequency bandwidth. However, due to the presence of hydrostatic pressure and sound pressure, the upper cavity is squeezed and deformed. Therefore, in the higher frequency bandwidth, the upper cavity contributes less to the sound absorption energy, so both the resonance and anti-resonance frequencies move to high frequencies. At this time, local resonance and wave-mode transformation sound absorption play a leading role.

The introduction of the metal vibrator and cavity into the rubber matrix forms a local resonance structure, which is equivalent to introducing a lowfrequency spring-mass mode, as shown in Fig. 2a. There, the steel backing and the bottom vibrator are equivalent to the external mass $M$, the upper vibrator is equivalent to the internal mass $m$, the composite matrix is equivalent to the spring, and the cavity provides compression space. When the frequency of the incident sound wave is close to the frequency of the mode, the sound wave and the structure have a strong coupling effect at $121 \mathrm{~Hz}$, as shown in Fig. 2b. The internal and external masses move in opposite directions. In addition, the upper rubber matrix and the inner mass $m$ have larger vibration displacements, while the middle and lower rubber displacements are smaller. In this case, the sound energy is converted into structural vibration energy, and the energy dissipation of the rubber damping effect realizes the sound absorption effect.

In the structure proposed in this paper, Young's modulus of the rubber is much smaller than Young's modulus of the vibrator, and the mass $m$ of the vibrator is very large, and its resonance frequency $\omega_{0}=\sqrt{k / m}$ can be quite low, where $k$ is the spring constant, and the resonance frequency is independent of the periodic element size [23]. Under

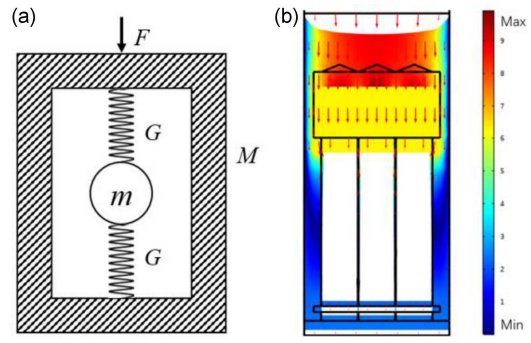

Fig. 2. (a) Simplified model of the soundabsorbing structure, (b) the total vibration displacement diagram $[\mathrm{mm}]$ at resonance frequency $(121 \mathrm{~Hz})$, the red arrow indicates the displacement vector.

the excitation of the elastic wave of a specific frequency, each scatterer resonates and interacts with the elastic wave, thereby suppressing the propagation of the elastic wave and generating a band gap. In the acoustic wave incident process, the resonance region of multiple cavities and matrix (see Fig. 1c and $\mathrm{d}$ ) is equivalent to introducing multiple local resonators into a lattice cell. Through the strong coupling between each oscillator, a new form of resonance is generated, which achieves a high sound absorption effect in a larger frequency range.

In addition, the propagation process of longitudinal waves along different media surfaces in this material is continuously transformed into shear waves. The rubber matrix is more prone to volume compression deformation, and the volume deformation around the interface of different media will be transformed into the shear deformation of the viscoelastic material. It is precisely due to the shear deformation at multiple different interfaces that the incident longitudinal wave is converted into an easily absorbing shear wave. The sound energy is rapidly attenuated in the viscoelastic rubber and converted into heat energy and dissipated, thereby increasing the internal friction, as shown in Fig. 1e and f. At the same time, the metal block acts as a vibrator in it. Its vibration in the rubber matrix not only directly affects the shape of the mold cavity, but also increases deformation amplitude of the matrix rubber in the $x, y$, and $z$ directions. The shear deformation increases the effective loss factor, which promotes the wave-mode transformation efficiency in the rubber matrix and improves the sound absorption performance.

\section{Structure design and simulation results}

\subsection{Structure description}

This paper presents an underwater soundabsorbing metamaterial for low-frequency bandwidth. Its three-dimensional and planar structure is shown in Fig. 3. Figure 3a is the basic structural unit. Two rectangular metal discs are inserted into the butyl rubber matrix material, and five cavities 
(a)
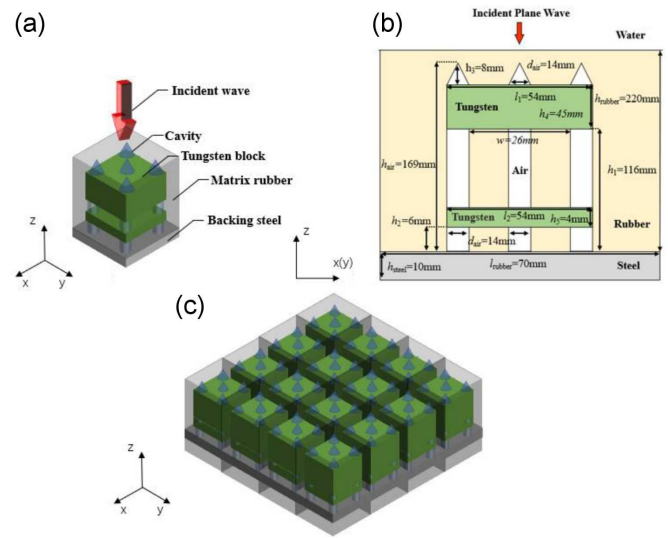

Fig. 3. Three-dimensional (a, c) and plane (b) structure of sound-absorbing materials. The white and green areas represent the cavity and the metal plate respectively. The geometric parameters for the best sound absorption coefficient are: $h_{\text {steel }}=10 \mathrm{~mm}$, $h_{\text {rubber }}=210 \mathrm{~mm}, l_{\text {rubber }}=70 \mathrm{~mm}, h_{\text {air }}=190 \mathrm{~mm}$, $d_{1}=d_{\text {air }}=25 \mathrm{~mm}, h_{1}=125 \mathrm{~mm}, h_{2}=6 \mathrm{~mm}$, $h_{3}=5 \mathrm{~mm}, l_{1}=l_{2}=61 \mathrm{~mm}, h_{4}=45 \mathrm{~mm}$, $h_{5}=4 \mathrm{~mm}, w=11 \mathrm{~mm}$.

of the same volume are divided into upper, middle, and lower parts. The upper cavities are cones and the middle and lower parts are cylinders. The steel backing is located under the rubber matrix material. The vibration of the square metal block in the rubber matrix not only directly affects the shape of the cavity, but also can cause more shear deformation, thereby indirectly improving the wavemode transformation efficiency in the rubber matrix. Figure $3 \mathrm{~b}$ shows a cross-section of the structural unit to more intuitively show the size of each constituent material. The parameters of each material are given in Table I.

\subsection{Simulation results}

In this paper, the acoustic-structure coupling module of COMSOL Multiphysics 5.4 is used to calculate the sound absorption coefficient. The upper and lower environments of this model are both water, and there are three boundary conditions. The boundary condition of the solid boundary is that the normal and tangential stresses are applied continuously on the solid and the solid boundary, and the displacement of the particle in each direction is continuous. The boundary condition of the solidwater boundary is: the normal stress in the solid is equal to the sound pressure in the water, but in the opposite direction; the normal particle displacement is continuous, and the tangential stress and displacement are both zero. The solid-air boundary is approximately a free interface, and the condition is that the normal stress and the tangential stress are equal to zero. The acoustic structure coupling module can handle the connection between the solid vibration and the sound field well.
Component material parameters.

TABLE I

\begin{tabular}{l|c|c|c|c}
\hline \hline Material & $\begin{array}{c}\text { Density } \\
{\left[\mathrm{kg} / \mathrm{m}^{3}\right]}\end{array}$ & $\begin{array}{c}\text { Acoustic } \\
\text { velocity } \\
{[\mathrm{m} / \mathrm{s}]}\end{array}$ & $\begin{array}{c}\text { Poisson's } \\
\text { ratio }\end{array}$ & $\begin{array}{c}\text { Young's } \\
\text { modulus } \\
{[\mathrm{GPa}]}\end{array}$ \\
\hline water & $1 \times 10^{3}$ & 1500 & - & - \\
air & 1.29 & 340 & - & - \\
tungsten & $1.935 \times 10^{4}$ & - & 0.28 & 360 \\
steel & $7.89 \times 10^{3}$ & - & 0.269 & 206 \\
rubber & $1.1 \times 10^{3}$ & - & 0.49 & -
\end{tabular}

We assume that the fluid is an ideal fluid, it has the characteristics of non-viscosity, uniformity and zero flow rate. Further, we assume that the density and pressure remain unchanged [17].

Fluid finite element equation is [24]

$$
\left[M^{p}\right]\{\ddot{p}\}+\left[K^{p}\right]\{p\}+\rho_{0}[R]\{\ddot{\delta}\}=\{\Phi\},
$$

where $\left[M^{p}\right],\left[K^{p}\right]$ and $[R]$ are the mass matrix, stiffness matrix, and coupling matrix of the whole fluid, respectively. The density of the fluid medium is $\rho_{0}$, $\{p\}$ is the sound pressure, $\{\delta\}$ is the displacement of the structural particle, and $\{\ddot{p}\}$ and $\{\ddot{\delta}\}$ are the second derivative of the sound pressure and displacement, respectively. Now, $\{\Phi\}$ represents the effect of the sound field in the outer unbounded fluid area on the fluid interface on the sound field in the finite fluid area [24]. Moreover, one can write that

$$
\left[M^{s}\right]\{\ddot{\delta}\}+\left[K^{s}\right]\{\delta\}=\left\{F^{s}\right\}+\left\{F^{p}\right\},
$$

where $\left\{F^{s}\right\}$ is the equivalent joint load of the structure under mechanical excitation, and $\left\{F^{p}\right\}$ is the equivalent joint load of the fluid acting on the structure. The overall stiffness matrix $\left[M^{s}\right]$ and the overall mass matrix $\left[K^{s}\right]$ of the solid structure are generated by the integration of the element stiffness matrix and the element mass matrix [24]

$$
\left\{\begin{array}{l}
{\left[M^{s}\right]=\sum_{e} \iiint_{V_{f}^{e}} \mathrm{~d} V\left[B_{\delta}\right]^{\mathrm{T}}[D]\left[B_{\delta}\right],} \\
{\left[K^{s}\right]=\rho_{s} \sum_{e} \iiint_{V_{f}^{e}} \mathrm{~d} V\left[N_{\delta}\right]^{\mathrm{T}}\left[N_{\delta}\right],}
\end{array}\right.
$$

where $V_{f}$ represents the fluid domain, $\left[B_{\delta}\right]$ is the strain matrix, $[D]$ is the elastic matrix reflecting the constitutive relationship of the material, $\rho_{s}$ is the structural material density, $\left[N_{\delta}\right]$ is the displacement interpolation shape function matrix, $V$ is the solid volume.

Combining the boundary conditions, one can deduce that

$$
\left\{F^{p}\right\}=\{R\}^{\mathrm{T}}\{p\} .
$$

The substitution of (4) into (3) provides the following equation [24]

$$
\left[M^{s}\right]\{\ddot{\delta}\}+\left[K^{s}\right]\{\delta\}-\{R\}^{\mathrm{T}}\{p\}=\left\{F^{s}\right\} .
$$

For the boundary condition $\{\Phi\}$ of the external boundary of the fluid domain, taking into account the incident, reflection and transmission of acoustic waves, the external boundary of the fluid at the incident end face and the transmission end face are 
written as $S^{-}$and $S^{+}$, respectively. In turn, the equivalent nodal loads of the sound pressure gradient at the incident end face and the transmission end face are written as $\left\{\Phi^{-}\right\}$and $\left\{\Phi^{+}\right\}$, respectively. Let

$$
\begin{aligned}
& {\left[C^{-}\right]=4 d_{1} d_{2}\left[A^{-}\right]^{\mathrm{T}}[D]\left[A^{-}\right]\left[C^{+}\right]=} \\
& 4 d_{1} d_{2}\left[A^{+}\right]^{\mathrm{T}}[D]\left[A^{+}\right],
\end{aligned}
$$

where $2 d_{1}$ and $2 d_{2}$ are the lengths of the unit structure in the $x$ and $y$ directions, respectively. The overall matrixes $\left[A^{-}\right]$and $\left[A^{+}\right]$are obtained by integrating the unit matrices

$$
\frac{1}{2 d_{1}} \cdot \frac{1}{2 d_{2}} \int_{S_{e}^{-}} \mathrm{d} S\left\{\mathrm{e}^{\mathrm{i}\left(k_{n x} x+k_{m y} y\right)}\right\}_{N M \times 1}\left\{N_{p}\right\}^{\mathrm{T}}
$$

and

$$
\frac{1}{2 d_{1}} \cdot \frac{1}{2 d_{2}} \int_{S_{e}^{+}} \mathrm{d} S\left\{\mathrm{e}^{\mathrm{i}\left(k_{n x} x+k_{m y} y\right)}\right\}\left\{N_{p}\right\}^{\mathrm{T}},
$$

where $k_{n m}$ represents the mode of each order of axial waves.

Now, $\{\Phi\}$ can be written as

$$
\begin{aligned}
& \{\Phi\}=\left\{\left\{\Phi^{+}\right\} \quad\{0\} \quad\left\{\Phi^{-}\right\}\right\}= \\
& \left\{\begin{array}{c}
2\left\{\Phi_{i}\right\} \\
\{0\} \\
\{0\}
\end{array}\right\}+\left[\begin{array}{ccc}
{\left[C^{-}\right]} & \{0\} & \{0\} \\
\{0\} & \{0\} & \{0\} \\
\{0\} & \{0\} & {\left[C^{+}\right]}
\end{array}\right]\{p\} .
\end{aligned}
$$

Let

$$
\left[C_{\Phi}\right]=\left[\begin{array}{ccc}
{\left[C^{-}\right]} & \{0\} & \{0\} \\
\{0\} & \{0\} & \{0\} \\
\{0\} & \{0\} & {\left[C^{+}\right]}
\end{array}\right]
$$

and $\left\{C_{0}\right\}=\left\{2\left\{\Phi_{i}\right\}^{\mathrm{T}},\{0\}^{\mathrm{T}},\{0\}^{\mathrm{T}}\right\}$ keep the sequence order of the fluid nodes corresponding to the elements in $\left[M^{p}\right],\left[K^{p}\right]$ and $[R]$. They are consistent with the sequence order of the fluid nodes corresponding to the elements in $\{\Phi\}$. Then the fluid-structure coupling finite element equation of the entire system is organized as

$$
\begin{gathered}
{\left[\begin{array}{cc}
-[R]^{\mathrm{T}} & {\left[K^{s}\right]-\omega^{2}\left[M^{s}\right]} \\
{\left[K^{p}\right]-\left[C_{\Phi}\right]-\omega^{2}\left[M^{p}\right]} & -\rho_{0} \omega^{2}[R]
\end{array}\right]\left\{\begin{array}{l}
\{p\} \\
\{\delta\}
\end{array}\right\}=} \\
\left\{\begin{array}{l}
\left\{F^{s}\right\} \\
\left\{C_{0}\right\}
\end{array}\right\} .
\end{gathered}
$$

The boundary conditions satisfied by the node displacement $\{\delta\}$ and the fluid node voltage $\{p\}$ of the periodic element structure are

$$
\left\{\begin{array}{l}
\left\{\chi_{S_{3}}\right\}=\left\{\chi_{S_{1}}\right\} \mathrm{e}^{\mathrm{i} \psi_{x}}, \\
\left\{\chi_{S_{4}}\right\}=\left\{\chi_{S_{2}}\right\} \mathrm{e}^{\mathrm{i}\left(\psi_{\Delta x}+\psi_{y}\right)}, \\
\left\{\chi_{C_{2}}\right\}=\left\{\chi_{C_{1}}\right\} \mathrm{e}^{\mathrm{i} \psi_{x}}, \\
\left\{\chi_{C_{3}}\right\}=\left\{\chi_{C_{1}}\right\} \mathrm{e}^{\mathrm{i}\left(\psi_{\Delta x}+\psi_{y}\right)}, \\
\left\{\chi_{C_{4}}\right\}=\left\{\chi_{C_{1}}\right\} \mathrm{e}^{\mathrm{i}\left(\psi_{x}+\psi_{\Delta x}+\psi_{y}\right)},
\end{array}\right.
$$

where $S_{1}, S_{2}, S_{3}, S_{4}$ refer to the boundary surface of the periodic unit, $C_{1}, C_{2}, C_{3}, C_{4}$ refer to the boundary line of the periodic unit, $\chi$ represents $\delta$ or $p$, and $\psi_{x}=-2 d_{1} k \sin (\theta) \cos (\varphi), \psi_{y}=$ $-2 d_{2} k \sin (\theta) \cos (\varphi), \psi_{\Delta x}=-(\Delta x) k \sin (\theta) \cos (\varphi)$.

In the fluid-solid structure equation, each matrix is first divided into blocks according to the nodes corresponding to the elements. Then, combined with the boundary conditions, (11) can be written as

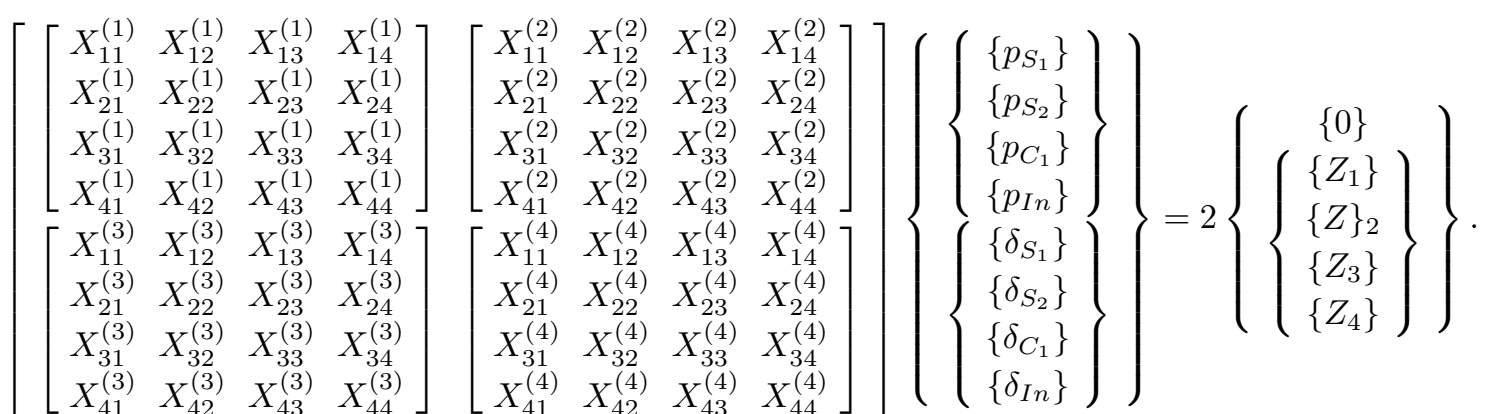

Let us take $N=\left(2 M_{x}+1\right)\left(2 M_{y}+1\right)$ nodes on the outer boundary of the fluid and establish $N$ equations for the relationship between node coordinates and sound pressure. By solving (13), the displacement and sound pressure of each node in the element can be obtained. The sound pressure of the reflected wave and incident wave on the boundary, $S^{-}$and $S^{+}$, are expressed with a finite number of steps in the form of a matrix, i.e.,

$$
\left\{\begin{array}{l}
p_{r}\left(x_{i}, y_{i}\right)=\sum_{n=-M_{x}}^{M_{x}} \sum_{n=-M_{y}}^{M_{y}} R_{n m}^{p} \mathrm{e}^{-\mathrm{i}\left(k_{n x} x_{i}+k_{m y} y_{i}-k_{n m} z_{-}\right)}=\left\{E_{r}^{(i)}\right\}_{1 \times N}\left\{R_{n m}^{p}\right\}_{N \times 1}, \\
p_{i}\left(x_{i}, y_{i}\right)=\sum_{n=-M_{x}}^{M_{x}} \sum_{n=-M_{y}}^{M_{y}} T_{n m}^{p} \mathrm{e}^{-\mathrm{i}\left(k_{n x} x_{i}+k_{m y} y_{i}-k_{n m} z_{+}\right)}=\left\{E_{t}^{(i)}\right\}_{1 \times N}\left\{T_{n m}^{p}\right\}_{N \times 1},
\end{array}\right.
$$



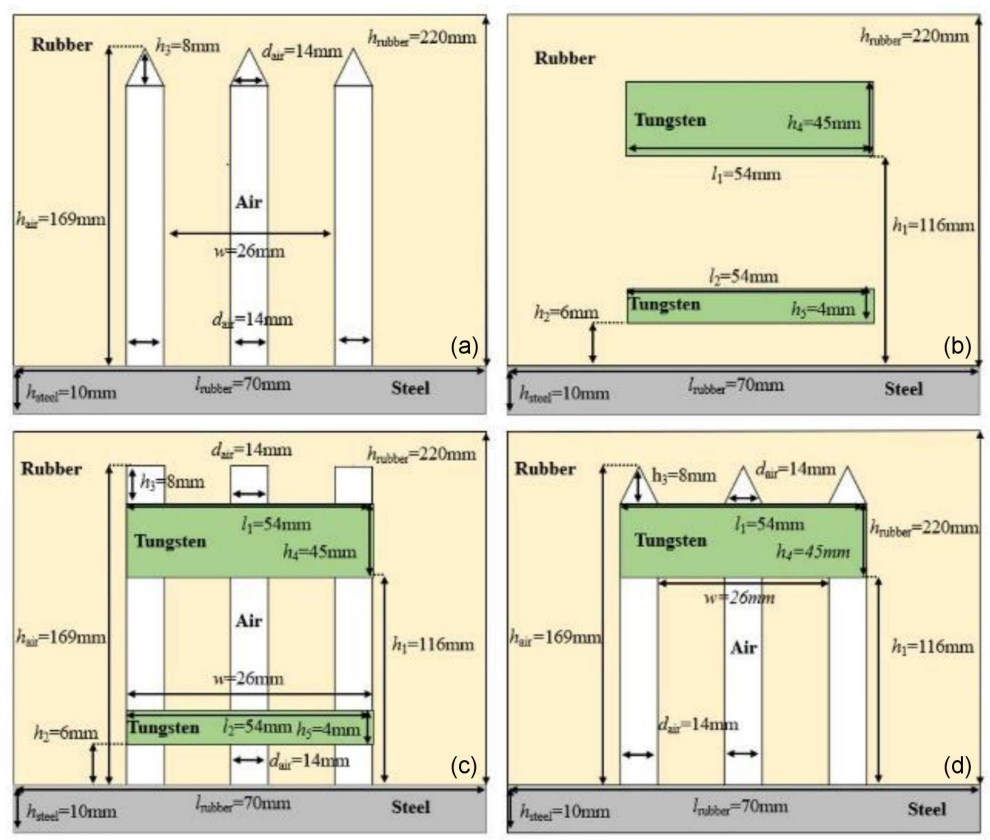

Fig. 4. Control group structure and parameters: (a) is not including the metal disk, (b) is the cavity on the cylinder.

where $i=1,2,3, \cdots N$. In $(14), p_{r}$ and $p_{t}$ are the reflected and transmitted sound pressure of nodes, respectively, $R_{n m}^{p}$ and $T_{n m}^{p}$ are the reflected and transmitted sound pressure amplitude of the $(n, m)$-order when the sound pressure amplitude of incident wave is the unit value, and

$$
\left\{\begin{array}{l}
\left\{E_{r}^{(i)}\right\}=\left\{\mathrm{e}^{-\mathrm{i}\left(k_{n x} x_{i}+k_{m y} y_{i}-k_{n m} z_{-}\right)}\right\}, \\
\left\{E_{t}^{(i)}\right\}=\left\{\mathrm{e}^{-\mathrm{i}\left(k_{n x} x_{i}+k_{m y} y_{i}-k_{n m} z_{+}\right)}\right\},
\end{array}\right.
$$

for $\left(n=-M_{x}: 1: M_{s}, m=-M_{y}: 1: M_{y}\right)$.

From the perspective of acoustic energy calculation, the reflection or transmission coefficient can be expressed as

$$
\left\{\begin{aligned}
r & =\frac{1}{N} \sqrt{\sum_{n} \sum_{m} R_{n m}^{2}} \\
t & =\frac{1}{N} \sqrt{\sum_{n} \sum_{m} T_{n m}^{2}}
\end{aligned}\right.
$$

Therefore, the expression of the sound absorption coefficient is

$$
\alpha=1-|r|^{2}-|t|^{2} .
$$

\section{Results and discussion}

\subsection{Comparison with similar structural models}

In order to highlight the high sound absorption performance of this material at low frequencies, we provide four control groups as a comparison, as shown in Fig. 4. Control group A (CG1) does not include the tungsten metal disks, control group $\mathrm{B}$ (CG2) has only two tungsten metal disks, the top cavities of control group C (CG3) are cylinders, and control group D (CG4) has only cavities and the upper tungsten metal disk. In addition, other geometric dimensions and material properties are the same as the present structure. The sound absorption performance comparison of the present structure and the above four structures between 0 and $300 \mathrm{~Hz}$ are shown in Fig. 5. It can be seen in Fig. 5b that the sound absorption effect of rubber with metal disks is very weak. The combination of cavities and metal disks can significantly improve the absorption coefficient, indicating the local resonance mechanism for absorption. After replacing the top cylinder cavities with the conical ones, the overall acoustic absorption coefficient between 140 and $300 \mathrm{~Hz}$ is increased, which results in the absorption mechanism of wave-mode transformation. The results comparison between the present structure and control group D shows that the addition of the vibrator can also improves the sound absorption performance between 140 and $300 \mathrm{~Hz}$. The sound absorbing coefficient of the present metamaterial reaches above 0.7 within 45 to $300 \mathrm{~Hz}$ bandwidth and remains more than 0.9 within 110 to $300 \mathrm{~Hz}$ bandwidth. At $100 \mathrm{~Hz}$, the thickness of the structure is only $1 / 68$ of the length of the acoustic wave in water, which proves that the structure has the characteristics of "small size control large wavelength" in an underwater environment.

\subsection{The influence of geometrical parameters on sound absorption performance}

Based on the above-mentioned optimal sound absorption coefficient structural parameter model, the influence of geometric parameters on the sound absorption performance between 0 and $300 \mathrm{~Hz}$ is analyzed. 


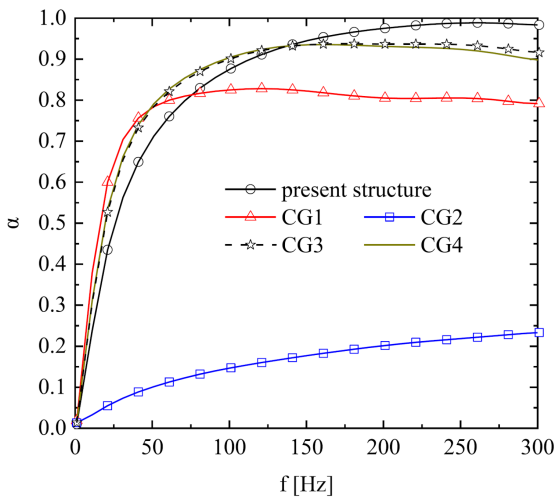

Fig. 5. Comparison of sound absorption coefficient between the present structure and the four control groups in the frequency range of $0-300 \mathrm{~Hz}$.

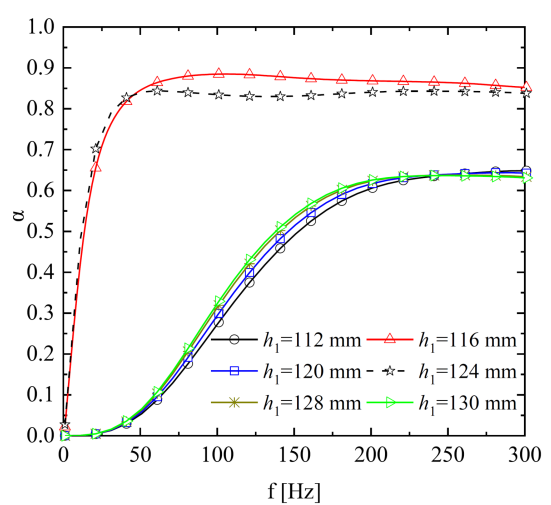

Fig. 6. The effect of changing the cavity height on the sound absorption coefficient.

Figure 6 shows the effect of cavity height $h_{1}$ on the sound absorption performance, which varies from 112 to $130 \mathrm{~mm}$. It can be seen that the specific cavity height significantly improves the sound absorption coefficient. When $h_{1}=116$ and $124 \mathrm{~mm}$, the average sound absorption coefficient of the structure in $300 \mathrm{~Hz}$ is the best, and it remains above 0.5 in the 21 to $300 \mathrm{~Hz}$ frequency bandwidth. The sound absorption coefficient reaches the peak value 0.88 at $100 \mathrm{~Hz}$ when the height $h_{1}$ is $116 \mathrm{~mm}$. It shows that as the height of the cavity increases, the relative position of the upper vibrator in the structure changes, and the relative volume of the upper rubber also gradually increases, causing the resonance and antiresonance frequencies to shift to high frequencies, thus affecting the low-frequency sound absorption effect. But the increase in the height of the cavity also increases the contact area between the cavity and the rubber matrix, which means that more shear deformation and sound wave scattering are introduced, and the wavemode transformation efficiency is improved. Therefore, $h_{1}=116$ and $124 \mathrm{~mm}$ show that the high sound absorption effect is balanced by the joint action of the local resonance and the wave-mode transformation mechanism.

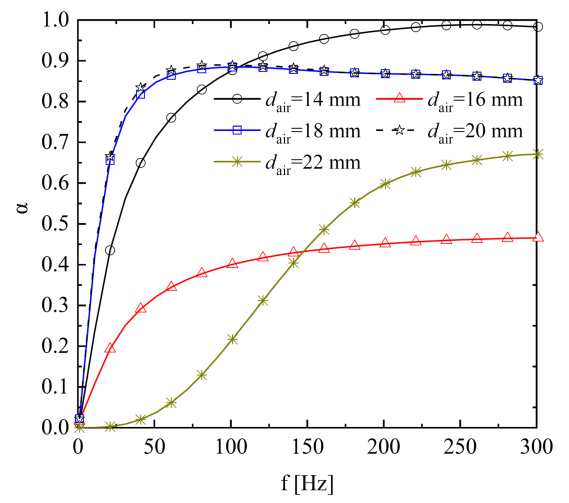

Fig. 7. The effect of changing the cavity diameter on the sound absorption coefficient.

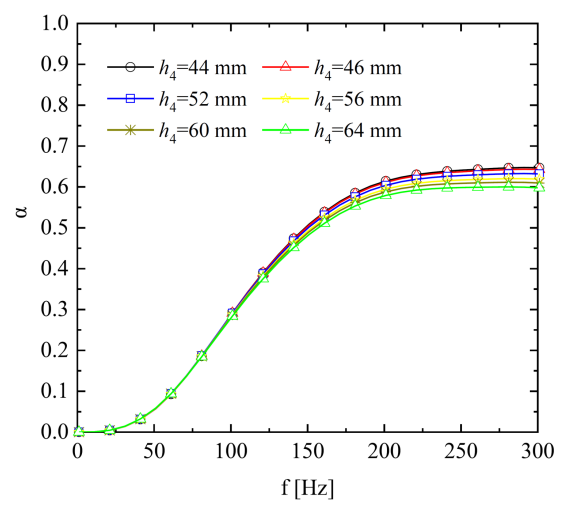

Fig. 8. The effect of the thickness of the upper vibrator on the sound absorption coefficient.

In addition, considering the influence of the cavity diameter, when $d_{\text {air }}$ increases from 14 to $22 \mathrm{~mm}$, the overall sound absorption effect continues to increase in the range of $20-300 \mathrm{~Hz}$, as shown in Fig. 7. When the diameter $d_{\text {air }}$ are 18 and $20 \mathrm{~mm}$, the sound absorption coefficient first increases and then decreases and tends to be stable. When $d_{\text {air }}=14 \mathrm{~mm}$, the sound absorption first increases, later it stabilizes. The overall absorption performance for $d_{\text {air }}=$ $14 \mathrm{~mm}$ is higher than that of $16 \mathrm{~mm}$. The sound absorption coefficient for $d_{\text {air }}=22 \mathrm{~mm}$ increases more rapid between 50 and $200 \mathrm{~Hz}$ than other frequencies. The reason for the above phenomena is that the larger the diameter $d_{\text {air }}$ in a certain range, the larger the volume of the cavity and the contact area with the rubber matrix, which means that the resonance frequency of the cavity moves to the lowfrequency as the volume increases, and at the same time under conditions, more wave-mode transformations will occur, transforming longitudinal waves into transverse waves that are easily absorbed by the rubber matrix, and improving the sound absorption coefficient.

Figure 8 shows the effect of the thickness of the upper vibrator on the sound absorption performance. As the thickness of the upper metal disc 
changes from 30 to $70 \mathrm{~mm}$, the structure exhibits different sound absorption effects, indicating that changing the thickness, that is, changing the mass of the metal vibrator, will cause the resonance frequency of the structure to change accordingly. When the thickness of the upper metal vibrator is $h_{4}=45$ and $60 \mathrm{~mm}$, the structure maintains high sound absorption performance in the 21 to $300 \mathrm{~Hz}$ frequency bandwidth. It is worth noting that when the thickness of the upper vibrator is $h_{4}=60 \mathrm{~mm}$, the sound absorption coefficient in 0 to $75 \mathrm{~Hz}$ is slightly higher than that of $h_{4}=45 \mathrm{~mm}$, indicating that the increase in the thickness of the upper metal vibrator as the internal mass will shift the resonance frequency to low frequencies. However, the sound absorption coefficient of $h_{4}=45 \mathrm{~mm}$ in the 75 to $300 \mathrm{~Hz}$ frequency bandwidth is higher than the former, reaching above 0.9 , indicating that the resonance frequency in this case is also higher than the former.

\section{Conclusion}

In this paper, a metal vibrator and a cavitycontaining rubber sound-absorbing material are combined, and based on the principles of local resonance, wave-mode transformation and scattering, an underwater sound-absorbing metamaterial suitable for 0 to $300 \mathrm{~Hz}$ is proposed. The results of the COMSOL software simulation show that the sound absorption coefficient of this material in the frequency range of 45 to $300 \mathrm{~Hz}$ can be maintained above 0.7 , and the best sound absorption coefficient can reach 0.99 . Finite element method analysis revealed the influence of different parameters on the sound absorption effect of the material under 0 to $300 \mathrm{~Hz}$, and explained that the combined effect of the local resonance, wave-mode transformation and sound wave scattering mechanism made a great contribution to the high sound absorption performance of the structure. These conclusions are of great significance to the design of low-frequency soundabsorbing units. This design has potential application value in using underwater acoustic structure to reduce acoustic reflectivity and realize underwater acoustic wave absorption.

\section{Acknowledgments}

This work was supported by the National Natural Foundation of China (Grants Nos. 11772349, 11972354 and 1972365).

\section{References}

[1] N. Gao, K. Lu, Appl. Acoust. 169, (2020). [2] J. Li, S. Li, J. Vibrat. Control 24, (2018).

[3] G.S. Sharma, A. Skvortsov, I. MacGillivray, N. Kessissoglou, Appl. Acoust. 143, 200 (2019).
[4] M.J. Sharifi, V. Ghalehkhondabi, A. Fazlali, J. Thermal Anal. Calorim. (2021) (prepublish).

[5] Z. Wang, Y. Huang, X. Zhang, L. Li, M. Chen, D. Fang, J. Sound Vibrat. 479, 115375 (2020).

[6] G. Moradi, P. Nassiri, A. ErshadLangroudi, M.R. Monazzam, Health Scope 8, e64862 (2019).

[7] G. Moradi, P. Nassiri, A. ErshadLangroudi, M.R. Monazzam, Plast. Rubb. Composit. 47, (2018).

[8] Z. Luo, X. Zhu, Z.T. Lin, W.Z. Wang, Ship Sci. Technol. 31, 23 (2009).

[9] W. Chen, S. Liu, L. Tong, S. Li, Theor. Appl. Mech. Lett. 6, 42 (2016).

[10] Yinghong Gu, Haibin Zhong, Bin Bao, Quan Wang, Jiuhui Wu, Appl. Acoust. 172, 107605 (2021).

[11] Mingyu Duan, Chenlei Yu, Fengxian Xin, Tian Jian Lu, Appl. Phys. Lett. 118, 071904 (2021).

[12] W.L. Tang, S.P. He, J. Fan, Acta Acust. 30, 289 (2005).

[13] P.A. Deymier, Acoustic Metamaterials and Phononic Crystals, Springer, Heidelberg 2013.

[14] M. Oudich, B. Djafari-Rouhani, B. Bonello, Y. Pennec, S. Hemaidia, F. Sarry, D. Beyssen, Phys. Rev. Appl. 9, 034013 (2018).

[15] M. Tao, J. Vibrat. Control 20, 339 (2014).

[16] G.S. Sharma, A. Skvortsov, I. MacGillivray, N. Kessissoglou, J. Acoust. Soc. America 141, 4694 (2017).

[17] D. Zhao, H. Zhao, H. Yang, J. Wen, Appl. Acoust. 140, 183 (2018).

[18] R. Zhang, Y. Song, H. Hou, N. Gao, Mod. Phys. Lett. B 35, 2150039 (2021).

[19] C. Zwikker, C.W. Kosten, Sound Absorbing Materials, Elsevier, New York 1949.

[20] Junyi Wang, Jiaming Hu, Yun Chen, Front. Mech. Eng. 7, (2021).

[21] Y. Chen, G. Li, R. Sun, G. Chen, Crystals 11, 774 (2021).

[22] Y.R. Wang, X.H. Miu, H. Jiang, M. Chen, Y. Liu, W.S. Xu, D. Meng, Adv. Mech. 47, 92 (2017).

[23] H.Y. Wang, M.P. Zhou, Y.H. Fan, Z.Y. Zhang, in: Proc. National Academic National Conf. on Environmental Acoustics Beihai, Guangxi (China), 2009.

[24] G.Q. Liu, J.J. Lou, Q.W. He, MATEC Web Conf. 77, 08002 (2016). 